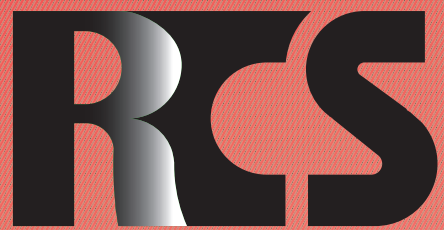

Depósito legal ppi $201502 Z U 4662$

Esta publicación científica en formato digital es continuidad de la revista impresa Depósito Legal: pp $197402 Z U 789$

- ISSN: 1315-9518 • ISSN-E: 2477-9431

Revista de Ciencias Sociales

Universidad del Zulia. Revista de la Facultad de Ciencias Económicas y Sociales Vol. XXVII. No. 2

Abril-Junio 2021

Esta publicación científica en formato digital es continuidad de la revista impresa Depósito Legal: pp $197402 Z$ Z789 ISSN: 1315-9518 


\title{
Modelo logístico para el aprovechamiento del potencial turístico del municipio Santo Tomás-Atlántico en Colombia
}

\author{
Pizarro, Ana* \\ Barrera, Alonso** \\ Ballestas, Marlene*** \\ Ramírez, Javier****
}

\section{Resumen}

Las dinámicas actuales que viven los espacios rurales llevan a la sociedad a repensar la forma en que son vistos estos territorios. Así, el objeto del estudio es diseñar un modelo logístico para el aprovechamiento del potencial turístico del municipio Santo Tomás-Atlántico en Colombia. Se desarrolla con un diseño de campo de tipo descriptivo. Se tomó una muestra representativa basada en un muestreo probabilístico estratificado; en total fueron consultadas 386 personas de la población asentada en el municipio y 14 funcionarios representantes de los entes gubernamentales, garantizándose la muestra mínima definida en el estudio. El análisis de toda la información obtenida permitió caracterizar el municipio de Santo Tomás (Atlántico) desde su potencial en turismo rural a partir de las condiciones actuales del territorio tanto físicas, ambientales, sociales, económicas como institucionales. A su vez, se plantea un nuevo modelo logístico para el aprovechamiento del potencial turístico del municipio Santo Tomás-Atlántico basándose en un equilibrio entre los productores directos y los intermediarios con la comunidad para fortalecer la cooperación. Se concluye, que el municipio de Santo Tomás (Atlántico) cuenta con vocación turística para desarrollar la actividad en el territorio, sustentada en paisajes con belleza escénica y alternativas de diversas actividades en ellas.

Palabras claves: Ecoturismo; modelo logístico; turismo rural; potencial turístico; Santo Tomás-Atlántico.

\footnotetext{
* Magister en Administración de Empresas e Innovación. Docente de la Institución Universitaria ITSA, Colombia. E-mail: ampizzaro1980@gmail.com iD ORCID: https://orcid.org/0000-0002-2901-8170

** Magister en Estadística. Docente de la Universidad de la Costa, Colombia. E-mail: abarrera@cuc.edu.co iD ORCID: https://orcid.org/0000-0001-5328-0454

*** Magister en Estadística. Docente de la Institución Universitaria ITSA, Colombia. E-mail: mballestas@itsa.edu.co iD ORCID: https://orcid.org/0000-0003-1546-1478

***** Estudiante del Programa Profesional en Administración Financiera de la Corporación Universitaria Latinoamericana, Colombia. E-mail: jramirez07papers@gmail.com iD ORCID: https://orcid.org/0000-0002-3663-9215
} 


\title{
Logistic model to take advantage of the tourism potential of the Santo Tomás- Atlántico municipality in Colombia
}

\begin{abstract}
The current dynamics that rural spaces are experiencing lead society to rethink the way these territories are viewed. Thus, the object of the study is to design a logistic model to take advantage of the tourism potential of the Santo Tomás-Atlántico municipality in Colombia. It is developed with a descriptive field design. A representative sample was taken based on a stratified probability sampling; In total, 386 people from the population settled in the municipality and 14 officials representing government entities were consulted, guaranteeing the minimum sample defined in the study. The analysis of all the information obtained allowed to characterize the municipality of Santo Tomás (Atlántico) from its potential in rural tourism from the current physical, environmental, social, economic and institutional conditions of the territory. At the same time, a new logistic model is proposed to take advantage of the tourism potential of the Santo Tomás-Atlántico municipality based on a balance between direct producers and intermediaries with the community to strengthen cooperation. It is concluded that the municipality of Santo Tomás (Atlántico) has a tourist vocation to develop the activity in the territory, supported by landscapes with scenic beauty and alternatives of various activities in them.
\end{abstract}

Keywords: Ecotourism; logistic model; rural tourism; tourism potential; Saint Thomas-Atlantic.

\section{Introducción}

Las dinámicas actuales que viven los espacios rurales en Colombia llevan a la sociedad a repensar la forma en que son vistos estos territorios, porque están creciendo en diferentes formas y realizando diferentes actividades económicas, tanto de transformación de bienes primarios como la prestación de servicios en diferentes ámbitos, y otras estrechamente ligadas con la naturaleza, como son el turismo y la recreación (Niebles, Santamaria y Niebles, 2018; Ascón y Argibay, 2020; Loor, Plaza y Medina, 2021; Nugra, et al., 2021). En particular, diversos estudios coinciden en que el turismo rural, es una potencial actividad económica alternativa para el desarrollo de esos territorios (Constabel, Oyarzun y Szmulewicz, 2008; Ministerio de Comercio, Industria y Turismo, 2012a; 2012b), a la vez que sugieren que sea combinado con otras actividades económicas que permitan generar ingresos y fuentes de empleo (Faiguenbaum, 2011; Morales, et al., 2015; Martín, et al., 2017).

Según la Organización Mundial del Turismo (OMT, 2017), el turismo es uno de los sectores que más aporta a las economías de los países. Es así, como Europa registró un 8\% más de llegadas internacionales en el año 2017 con respecto al año anterior; por su parte, los países africanos presentaron un crecimiento del $8 \%$, la región asiática y del Pacífico, evidenció un crecimiento del $6 \%$, el Oriente Medio del 5\% y el continente americano del 3\%. Asimismo, Zúñiga-Collazos (2015), describe que, para el caso específico del desarrollo turístico en Colombia, los resultados han sido positivos con un crecimiento del flujo turístico internacional de $+3,6 \%$ entre los años 2012-2013, logrando obtener ingresos muy importantes por este concepto.

Ciertamente, se debe mencionar como este sector ha cobrado una gran importancia en los últimos años (Diaz-Christiansen, Pérez-Gálvez y Ortega, 2017). Destaca un 
aumento significativo en lo que respecta a los procesos de planificación y puesta en marcha de los eventos que generan una mayor cantidad de beneficios, haciendo necesario el aprovechamiento de recursos para satisfacer a los consumidores (Gadotti y Stock, 2009).

Es aquí, donde aparece la logística como una herramienta de trabajo para ser aplicada en este tipo de procesos, ello ciertamente requiere de un análisis completo de las características y el potencial turístico de las locaciones, de manera que esta se adapte a la realidad de cada caso; integrándose entonces como pieza clave del turismo en la actualidad (Correa, Hernández y Loredo, 2017).

De este modo, a raíz de las crecientes tendencias del turismo a nivel mundial, Colombia en los últimos años, se ha posicionado como una opción interesante para quienes buscan alternativas diversas; convirtiéndose en una opción para un segmento importante de turistas nacionales y extranjeros, cuyo número aumenta día a día, y que disfrutan cada vez más de la amplia oferta de estos servicios (Niebles, et al., 2018; Martínez y Padilla, 2020).

Dentro de los estudios desarrollados sobre el sector turismo en el Departamento del Atlántico(Colombia), se evidencia la necesidad de profundizar indagaciones en relación a las potencialidades del turismo rural, debido a que, parte de estas investigaciones se concentran en cuantificar atractivos turísticos, midiéndose por ejemplo en términos de beneficios socioculturales y económicos en relación a las festividades locales, actividades desarrolladas por temporalidades, tal y como se presentan en la disertación de Gallardo (2009). Algunas investigaciones no llegan a profundizar en otros atractivos no estacionarios, que pueden redundar en los mismos o mejores beneficios y que, adicionalmente, pueden combinar de manera armónica los principios de tipo ambiental, social, cultural y económico.

Enlazando la realidad del sector turismo rural en el departamento del Atlántico con los procesos de logística aplicados en diversos contextos, se logra percibir una gran oportunidad para el desarrollo del potencial turístico de los municipios de dicho departamento, por medio de una metodología que se adapte a la realidad local y permita generar un elevado nivel de competitividad, frente a los procesos y dinámicas actuales, que se caracterizan por su complejidad y velocidad. Partiendo de ello, el presente estudio buscó diseñar un modelo logístico para el aprovechamiento del potencial turístico del municipio Santo Tomás-Atlántico en Colombia.

\section{Metodología}

Se contempla una investigación de campo, con el fin de revisar las características del municipio desde su potencial en turismo rural, consultando actores claves a través de encuestas cerradas. Por último, se desarrolla una investigación de índole aplicada para la propuesta del modelo presentado en la investigación.

Asimismo, en la investigación se aplicó un muestreo probabilístico estratificado, teniendo en cuenta que se consultó por segmentos dentro de los actores clave ligados al sistema turístico, observado para el municipio de Santo Tomás (Atlántico), considerando en cada grupo de ellos un universo de estudio. Para determinar el tamaño de la muestra, se tomó en cuenta a Spiegel y Stephen (2005), estableciendo para este estudio: $\mathrm{N} 1($ Población $)=23.188$ personas; un tamaño de $\mathrm{n} 1=386$ personas, y para N2 $($ Entes gubernamentales $)=18$ funcionarios; un tamaño de $\mathrm{n} 2=17$ funcionarios (representantes de entes gubernamentales a nivel local o departamental con alcance de sus funciones al municipio de Santo Tomás).

Se diseñó un instrumento, el cual fue aplicado sobre la muestra de manera escrita a través de medios electrónicos, permitiendo obtener la información para establecer, en conjunto con la información de tipo documental, la caracterización del potencial turístico rural del municipio de Santo Tomás (Atlántico). En total se utilizaron como instrumentos, dos (2) tipos de cuestionarios: 
Encuesta 1, dirigida a la población ubicada en el casco urbano y rural del municipio, que permitió obtener los datos necesarios sobre su grado de conocimiento y apreciación frente al desarrollo de la actividad turística rural del municipio; y Encuesta 2, dirigido a representantes de entes gubernamentales del nivel local y departamental. Esta permitió obtener los datos necesarios por parte de estos actores sobre acciones de promoción, fomento, visibilidad y apreciación, de la actividad turística del municipio, así como su potencial para el desarrollo del turismo rural.

Para efectos de la investigación, los instrumentos de recolección de datos fueron validados por expertos en su aspecto metodológico, específico en el área de turismo y finalmente estadístico. Respecto a la validación estadística, el instrumento (Encuesta 1 y 2) fue sometido en una primera instancia, a prueba de pilotaje con el $20 \%$ del tamaño de la muestra, es así como el piloto de la Encuesta 1, fue aplicado a 76 personas y la Encuesta 2, a 3 funcionarios; y en segunda instancia, fue aplicado el instrumento validado de acuerdo al tamaño de la muestra estimada, para la Encuesta 1, un total de 386 personas y para la Encuesta 2, un total de 14 de funcionarios.

Partiendo de ello, se pasa a una investigación aplicada en la segunda fase del articulo; donde se conjugan propuestas de diversas investigaciones previas con la realidad contemplada para el diseño de un modelo logístico para el aprovechamiento del potencial turístico del municipio Santo TomásAtlántico en Colombia.

\section{Resultados y discusión}

\subsection{Parte: Trabajo de campo}

Además de ser necesario que el territorio posea características turísticas favorables, es importante conocer la opinión y percepción de sus habitantes, así como los actores que tienen incidencia en la toma de decisiones que permitan fomentar la actividad turística en el municipio. De forma general en los resultados se evidencia consenso social, es decir no se encuentran diferencias significativas que tiendan a dividir la percepción de los encuestados. Se presenta entonces, los hallazgos obtenidos de las encuestas aplicadas a los dos (2) segmentos de la población que hicieron parte de la muestra. Se iniciará con una caracterización de cada muestra, seguidos del análisis de las variables estudiadas en ellas.

En primera instancia, con relación a la caracterización de la población del municipio, en su gran mayoría esta se concentra en el casco urbano del municipio, el 97\% de los encuestados, en los sectores residenciales del municipio. El 3\% restante, hace referencia a la población concentrada en parcelas ubicadas en la zona rural, estos resultados evidencian el comportamiento demográfico actual del municipio, pues quienes habitan en la zona rural no supera el 5\% de la población total de Santo Tomás. Proyecciones del Departamento Administrativo Nacional de Estadística de Colombia (DANE, 2016), presentaban como problemática la migración de la población del área rural, la cual significaría disminución de habitantes en la zona rural de Santo Tomás cercana al $30 \%$.

La población es principalmente joven (entre 18 a 39 años) representada en un $58 \%$, en relación con la población adulta; el $18 \%$ de la población oscila entre los 40 a 50 años, un $19 \%$ con edades entre los 51 a 61 años, y en menor proporción se encuentran adultos-mayor superando los 60 años, representados por el $6 \%$ del total de la población consultada. La situación descrita da cuenta sobre la juventud de la población, quienes en gran medida tendrían por delante un enorme desafío: Ser conductores de un proceso de desarrollo económico y social en su municipio. La relación de géneros en el municipio se encuentra equilibrada, hombres con un $51 \%$ y las mujeres con un $49 \%$ entre los encuestados. Situación que puede representar en el municipio, la generación de oportunidades en igualdad de condiciones para cada uno de ellos.

Asimismo, la población del municipio, 
Pizarro, Ana Barrera; Alonso Ballestas y Marlene Ramírez, Javier

Modelo logístico para el aprovechamiento del potencial turístico del municipio Santo Tomás-

Atlántico en Colombia

cuenta con un nivel de educación que le permite desempeñarse en el entorno productivo, o la generación de oportunidades de negocio. El $74 \%$ de la misma, cuenta con formación técnico laboral, lo que evidencia que están formados y preparados para ocupaciones específicas; asimismo el 9\% de la población posee títulos profesionales de pregrado o postgrado, alcance que les permitirá analizar y participar en proyectos e iniciativas que promuevan el desarrollo de su territorio; el $17 \%$ de la población cuenta con formación a nivel de educación primaria o secundaria.

De igual manera, la encuesta reflejó que el $70 \%$ de la población se encuentra desempleada, un $28 \%$ se halla empleada, $\mathrm{y}$ un $2 \%$ desarrolla actividades de forma independiente. Esto evidencia la necesidad en la población de encontrar diversas fuentes de generación de ingresos, con la finalidad de contribuir al sostenimiento de sus familias y al crecimiento del municipio, así como en la mejora de su calidad de vida.

Seguidamente, sobre la caracterización de funcionarios entes gubernamentales, se tuvo el $94 \%$ de la participación de funcionarios de entes gubernamentales públicos del municipio de Santo Tomás, representados en los 13 honorables miembros del Consejo municipal, así como el señor Alcalde del municipio, el secretario de gobierno, el secretario de planeación y el Coordinador de cultura; y la participación del $6 \%$ de funcionarios del orden departamental, representado en el Subsecretario de Turismo del departamento del Atlántico.

Al indagar sobre la percepción de la calidad de vida en el municipio, las respuestas fueron positivas frente a la satisfacción de los habitantes de Santo Tomás (88\%); una parte de la población no logra precisar si está conforme frente a las condiciones de desarrollo local del municipio y a su nivel de calidad de vida; otros integrantes la calificaron como no satisfactoria (4\%). Las inconformidades, tienen relación con deficiencias en vías de acceso a la zona rural, y disponibilidad de servicios de energía eléctrica. Frente a la sostenibilidad, el $94 \%$ de la población afirma que, a través del ejercicio del turismo rural, el municipio fortalecerá su desarrollo, el 5\% manifiesta que este podría contribuir medianamente, y solo el $1 \%$ considera que la actividad turística no aportará al desarrollo de Santo Tomás.

$\mathrm{Al}$ consultar a los gobernantes su percepción del grado de desarrollo y calidad de vida alcanzado en el municipio actualmente, el $44 \%$ de los funcionarios se siente satisfecho, sin embargo, el $46 \%$ restantes manifiesta, en iguales proporciones, su insatisfacción o no tener certeza de esta. Por lo anterior, se evidencia como oportunidad para estos colegiados encontrar alternativas que permitan que el territorio mejore las condiciones de vida para su población, la visibilidad del municipio a nivel departamental, regional y nacional, asimismo, el fortalecimiento de la infraestructura tangible e intangible.

De igual forma, se encontró que solo el $44 \%$ de los funcionarios, manifiesta conocer lo que el municipio desarrolla en materia de turismo; por su parte el 17\% expresó estar al tanto parcialmente de las actividades turísticas que se realizan; y un 39\% manifiesta no tener conocimiento de dichas actividades. Durante el ejercicio, se pudo constatar que aun así participen de algunas actividades en esta localidad, tales como realizar paseos a fincas, participar en fiestas y tradiciones, estas no son consideradas como actividades de turismo.

En este mismo sentido, la mitad de los funcionarios encuestados declaró no tener conocimiento sobre la existencia de programas o planes para el desarrollo del turismo rural en el municipio, originados desde los órganos de gobierno local o departamental. No obstante, el 39\% manifestó estar al tanto de algunas iniciativas que propenden por fomentar el turismo hacia el municipio, estás son: La Ruta Religiosa, el Reinado Intermunicipal y Carnavales de Santo Tomás, contando con asignación presupuestal para su realización. Un $11 \%$ expresó no estar seguro de si existen o no algún tipo de plan o iniciativa para el fomento de la actividad turística del municipio.

Lo evidenciado representa una amenaza para el impulso de las iniciativas de desarrollo de turismo rural para el municipio, se reafirma el 
desconocimiento por parte de los funcionarios sobre el alcance que algunas actividades con tradición y trayectoria en el territorio, deben ser conocidas como actividades turísticas.

A través de la revisión documental, fue posible identificar que el municipio de Santo Tomás actualmente carece de un plan o programa para promocionar sus atractivos turísticos, evidenciándose por su no vinculación en el Plan de Desarrollo 2016-2019. Por parte de la Gobernación del Atlántico (2016), a través de la subsecretaría de turismo, se referencia solo una Ruta turística en la temporada de Semana Santa para disfrutar de los diferentes eventos culturales y religiosos que ofrecen varios municipios del departamento, entre los que se encuentra el municipio de Santo Tomás. Esta situación se refleja como una de las principales amenazas en el municipio, frente a su potencialidad para el desarrollo del turismo rural.

En cuanto a las potencialidades del municipio de Santo Tomás, en el caso de las actividades tradicionales, festivas y típicas, el $96 \%$ de la población coincidió que esta es su mayor fortaleza; seguido por la belleza escénica del municipio, representada en el patrimonio cultural, material inmueble $\mathrm{y}$ mueble, dispuesto dentro de su casco urbano, y los sitios naturales para desarrollar actividades inmersas en la zona rural del municipio con un 40\%; acompaña a esta actividad el ejercicio de caminatas y senderismo dentro de los espacios rurales con un 14\%; y finalmente, consideran que cuentan con una infraestructura y equipamiento que da soporte a turistas que lleguen al municipio, tales como restaurantes, hoteles, hospedajes en casa pobladores, teatro, hospital, almacenes, centros comerciales, entre otros, este aspecto considerado como fortaleza está representado en un $6 \%$.

Con relación a los atractivos turísticos del municipio, el $56 \%$ de los funcionarios encuestados afirma que, sí es posible con las condiciones actuales desarrollar actividades de tipo rural en Santo Tomás; mientras que el 28\% manifiesta medianamente estar de acuerdo frente a este supuesto; y un $17 \%$ de los encuestados, afirman que no existen tales potenciales en el municipio. A partir de estos resultados, se evidencia la necesidad de capacitar a los miembros de entes gubernamentales del nivel local sobre la temática, sus implicaciones, impactos y ventajas, frente a las nuevas políticas de gobierno de impulsar a través de la economía naranja sectores de la industria como lo es el turismo.

El consenso entre la población, se orienta hacia la falta de capacitación para recibir y tratar adecuadamente a los turistas, representado en un $76 \%$; seguido por las debilidades en mejores infraestructuras, tipo vías de acceso y disponibilidad de servicios de energía eléctrica en la zona rural, representado con un $29 \%$; un bajo porcentaje de la población, manifiesta que no habría mucho interés a la hora de hacer inmersión en la promoción y ejercicio de las actividades de turismo rural, representado en un $5 \%$; y finalmente solo un $1 \%$, considera que los turistas que lleguen al municipio poco sería de su interés en interactuar con los paisajes naturales y la vida de campo.

Los funcionarios, comparten con la población algunos aspectos identificados como debilidades del municipio; la falta de capacitación para recibiry trataradecuadamente a los turistas, representado en un $44 \%$, seguido de debilidades en infraestructuras, tipo vías de acceso y disponibilidad de servicios de energía eléctrica en la zona rural, representado con un $33 \%$; comparten el $22 \%$ restante en igual proporción, el no interés de la población en hacer inmersión en la promoción y ejercicio de las actividades de turismo rural, representado en un $11 \%$; y finalmente consideran que el municipio no cuenta con atractivos turísticos el $11 \%$ restante.

En cuanto a las oportunidades que brindan los atractivos turísticos del municipio, la población coinciden en un $89 \%$ en que los eventos de tradiciones, fiestas y actividades típicas del territorio, serían el principal atrayente; seguidamente aparecen con un $29 \%$ cada una, actividades como talleres de construcción de artesanías y el ejercicio de senderismo y caminata; la gastronomía típica, rica en sus sabores exóticos y ambientados 
en zonas de restaurantes en el área rural, representado con un 16\%; aparece como una nueva alternativa, la pesca artesanal, la cual puede desarrollarse en los humedales, ciénagas, lagos y ríos que se encuentran en el municipio, coinciden un $13 \%$ de la población; el realizar inmersión en las costumbres del pueblo o de sus zonas rurales, participan con un $12 \%$ de ser escogida esta actividad; finalmente, la interacción con actividades agrícolas, representa un $9 \%$ dentro de las opciones.

En relación a la percepción de las oportunidades del municipio para el ejercicio del turismo en él; por parte de los funcionarios, el $89 \%$ percibe que esta actividad es una alternativa de desarrollo. Sin embargo, existe un $11 \%$ que muestra resistencia frente a esta iniciativa. En el caso de la población, el 94\% percibe que esta actividad podría convertirse en una alternativa de desarrollo para el municipio, y sólo el $1 \%$ concibe que no aportaría. Es clara la evidencia al contrastar el resultado de estas variables con los hallados en la población, que estos últimos ofrecen mayor confianza, interés y disposición frente al desarrollo del turismo rural en el municipio de Santo Tomás.

A continuación, se presenta un análisis de cruce de variables y aspectos que caracterizan a la población a fin de conocer la real intencionalidad frente al ejercicio de la actividad turística rural:

Los resultados encontrados en la Tabla 1 sobre el turista, denotan que en la población a nivel general existe buena relación con turistas que llegan al municipio y que su presencia les agrada $(89,9 \%)$, de otra parte, en menor proporción la población considera que no existe tal relacionamiento con turistas $(8,3 \%)$ y en contraste con los resultados anteriores un bajo por ciento de la población $(1,8 \%)$ consideraría mejor que no estuviesen turistas en el municipio. Sobre este último aspecto, llama la atención que son los pobladores con educación básica y media, quienes rechazan la presencia de turistas en Santo Tomás.

Tabla 1

Tabla cruzada relación con turistas $\mathbf{x}$ nivel de estudio de la población

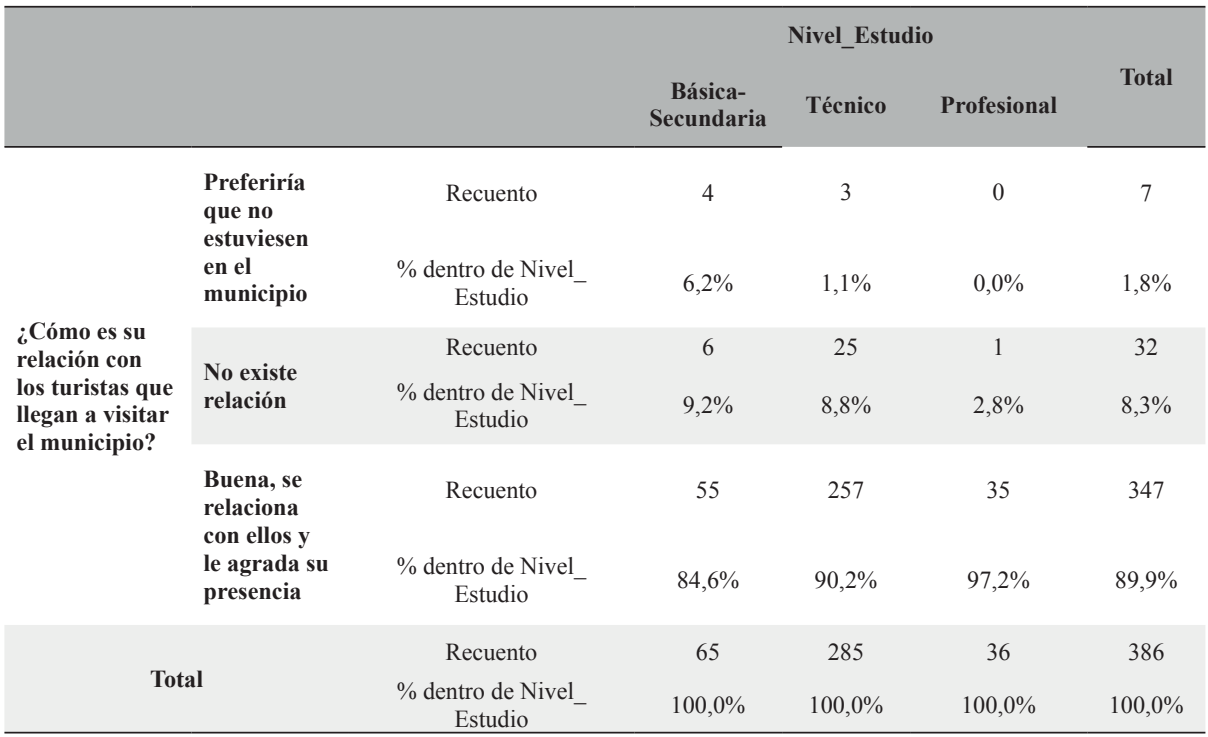

Fuente: Elaboración propia, 2020. 
Al considerar en la Tabla 2 el valor estadístico Chi-cuadrado de 10,203, la razón de verosimilitud de 8,856 , valores que difieren notablemente de 0 ; y nivel de significancia de 0,037 , inferior al nivel de confianza definido para este estudio; se evidencia que existe una dependencia entre las variables. Sin embargo, al analizar los resultados del cruce de variables en la Tabla 1, se logra concluir que aquellos con mayor nivel de estudios (Profesional 97,2\%; Técnico; 90,2\%) tendrán mayor disposición a relacionarse con los turistas que lleguen a su municipio, esto en contraste con la población que cuenta con estudios hasta nivel de secundaria $(84,6 \%)$.

Tabla 2

Pruebas Chi-cuadrado cruce de variables relación con turistas $\mathbf{x}$ nivel de estudio de la población

\begin{tabular}{lccc}
\hline & Valor & gl & $\begin{array}{c}\text { Significación asintótica } \\
\text { (bilateral) }\end{array}$ \\
\hline Chi-cuadrado de Pearson & $10,203^{\mathrm{a}}$ & 4 &, 037 \\
Razón de verosimilitud & 8,856 & 4 &, 065 \\
N de casos válidos & 386 & & \\
\hline
\end{tabular}

Nota: a. 3 casillas $(33,3 \%)$ han esperado un recuento menor que 5 . El recuento mínimo esperado es ,65.

Fuente: Elaboración propia, 2020.

De la misma manera, se refleja en la Tabla 1 que, los pobladores con mejor nivel de estudios (Profesionales 0,0\%; Técnico $1,1 \%$ ) no comparten la posibilidad que no estuviesen turistas en su territorio. Resulta entonces valido afirmar en esta investigación, que entre mayor sea el nivel de formación y conocimiento entre los pobladores, mayor su capacidad de aceptación y relacionamiento con turistas.

En equivalencia con el análisis anterior, en la Tabla 3 la población ya sea de zona rural (75\%) o del casco urbano $(90,4 \%)$, manifiestan relacionarse bien con los turistas. Frente a la posición de no querer relacionarse, llama la atención que esta condición solo se presenta en habitantes del casco urbano $(1,9 \%)$, para aquellos quienes habitan en el campo y zonas campestres no muestran indicios de no querer relacionarse con ellos $(0,0)$; situación que evidencia su interés en que las actividades de turismo rural puedan desarrollarse. Asimismo, al contrastar el recuento de datos de la Tabla 1 y 3 , permite inferir que el nivel de formación de la población rural le proporciona elementos suficientes para tener mayor receptividad frente a los resultados que podría generar un buen relacionamiento con los turistas interesados en los atractivos rurales. 


\section{Tabla 3}

Tabla cruzada relación con turistas $x$ zona donde habita la población

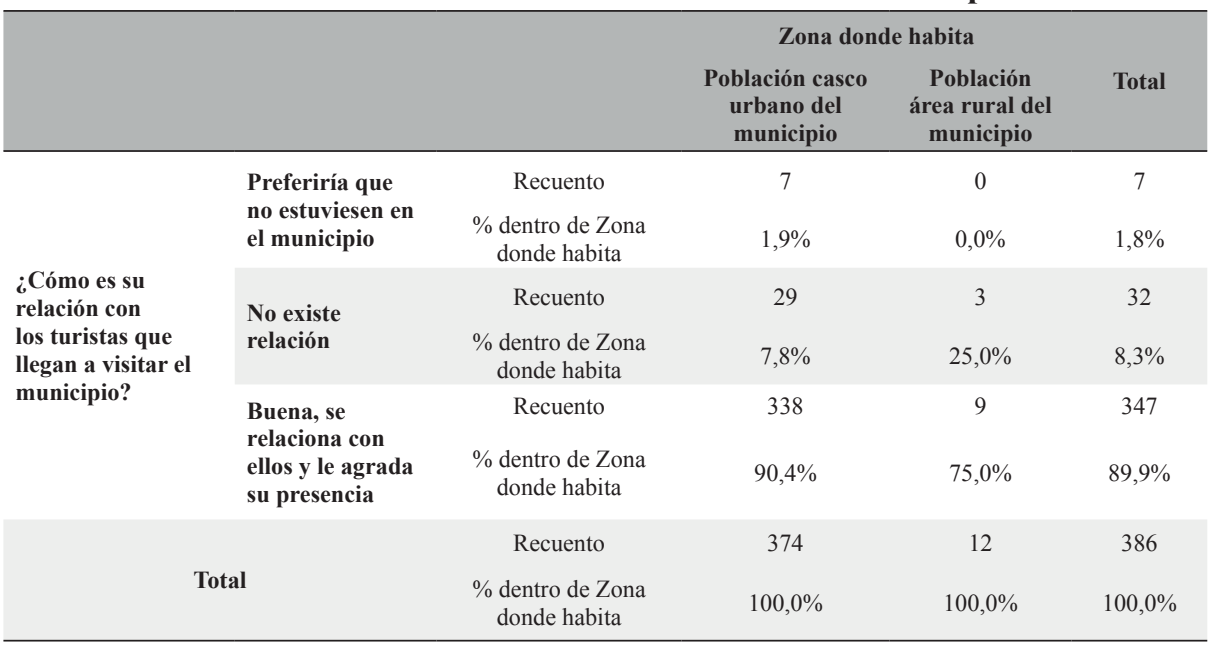

Fuente: Elaboración propia, 2020.

Al considerar si existe alguna relación con los turistas (femeninas 91,5\%; correspondencia entre el género de la masculinos 89,9\%). Por otra parte, se observa población y su relacionamiento con los turistas, una leve tendencia en el género masculino en puede concluir en la Tabla 4, que no existe manifestar preferir no relacionarse con turistas distinciones en cuanto al género sobre la buena $\quad(2,5 \%)$ frente a las mujeres $(1,1 \%)$.

Tabla 4

Tabla cruzada relación con turistas $\mathrm{x}$ género

\begin{tabular}{|c|c|c|c|c|c|}
\hline & & & & & \\
\hline & & & $\mathbf{F}$ & M & \\
\hline & Preferiría que no & Recuento & 2 & 5 & 7 \\
\hline Cómo es su & municipio & $\%$ dentro de Sexo & $1,1 \%$ & $2,5 \%$ & $1,8 \%$ \\
\hline relación con & & Recuento & 14 & 15 & 29 \\
\hline llegan a visitar el & & $\%$ dentro de Sexo & $7,4 \%$ & $7,6 \%$ & $7,5 \%$ \\
\hline тunipipio? & Buena, se relaciona & Recuento & 172 & 178 & 350 \\
\hline & su presencia & $\%$ dentro de Sexo & $91,5 \%$ & $89,9 \%$ & $90,7 \%$ \\
\hline & & Recuento & 188 & 198 & 386 \\
\hline & & $\%$ dentro de Sexo & $100,0 \%$ & $100,0 \%$ & $100,0 \%$ \\
\hline
\end{tabular}

Fuente: Elaboración propia, 2020. 
Es evidente considerar, que tal como se aprecia en la Tabla 5, no existe dependencia entre el género y su relacionamiento con los turistas, estadísticamente se corrobora al observar el nivel de significancia de 0,460, superior al nivel de confianza; rechazando alguna consideración de dependencia en la variable.

Tabla 5

Pruebas Chi-cuadrado cruce de variables relación con turistas $\mathbf{x}$ género

\begin{tabular}{lccc}
\hline & Valor & gl & $\begin{array}{c}\text { Significación asintótica } \\
\text { (bilateral) }\end{array}$ \\
\hline Chi-cuadrado de Pearson & $1,554^{\mathrm{a}}$ & 2 &, 460 \\
Razón de verosimilitud & 1,596 & 2 &, 450 \\
N de casos válidos & 386 & & \\
\hline
\end{tabular}

Nota: a. 2 casillas $(33,3 \%)$ han esperado un recuento menor que 5 . El recuento mínimo esperado es 3,41 .

Fuente: Elaboración propia (2020).

Un representativo porcentaje de población, estaría interesado en desarrollar actividades relacionadas con la promoción del turismo rural, solo fines de semana y días festivos $(59,8 \%)$ y de ellos se encuentra gran participación tanto de personas empleadas $(61,8 \%)$, como es de suponer para las personas que no cuentan con un empleo formal, quienes ven esta actividad como una alternativa de generación de ingresos $(55,2 \%)$, asimismo los que realizan actividades de forma independiente $(42,9 \%)$, como se evidencia en la Tabla 6. Dedicación completa en la semana de lunes a domingo (14,2\%), se ve orientada principalmente para aquellos pobladores que actualmente no cuentan con un empleo $(22,9 \%)$. Llama la atención de igual forma, que existe un porcentaje representativo $(25,9 \%)$ de la población, que realmente no estaría interesado en ejercer actividades turísticas.

\section{Tabla 6}

Tabla cruzada interés en la actividad turística $\mathbf{x}$ dedicación

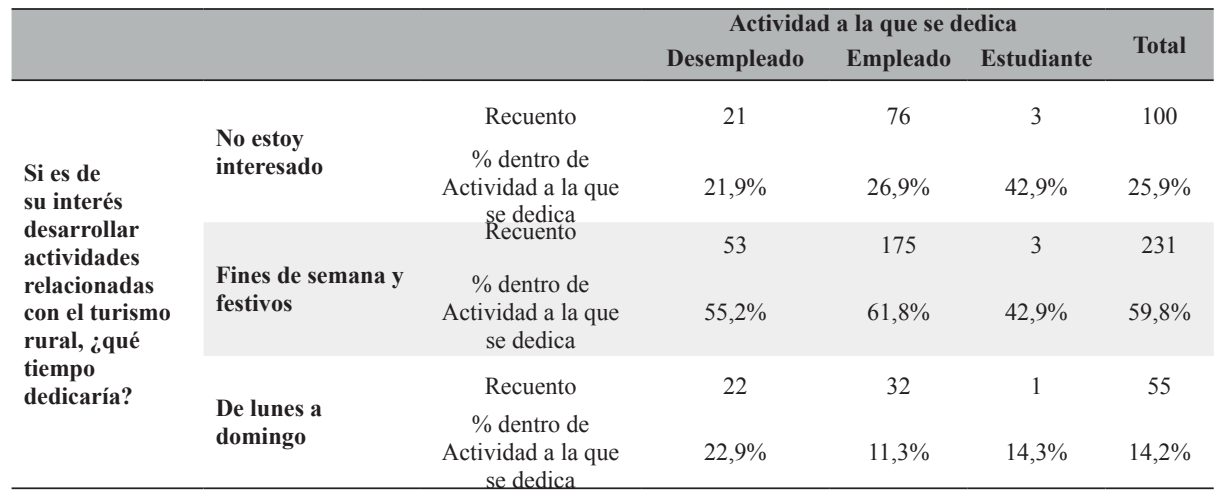




\section{Cont... Tabla 6}

\begin{tabular}{cccccc}
\hline & $\begin{array}{c}\text { Recuento } \\
\text { \% dentro de } \\
\text { Total }\end{array}$ & 96 & 283 & 7 & 386 \\
& $\begin{array}{c}\text { Actividad a la que } \\
\text { se dedica }\end{array}$ & $100,0 \%$ & $100,0 \%$ & $100,0 \%$ & $100,0 \%$ \\
\hline
\end{tabular}

Fuente: Elaboración propia, 2020.

Existe una relación entre el interés de la población en las actividades turísticas y el tiempo que emplearía en correspondencia con su ocupación. Estadísticamente se evidencia en la Tabla 7 esta dependencia, al observar el nivel de significancia de 0,048 inferior al nivel de confianza $(0,05)$.

Tabla 7

Pruebas Chi-cuadrado cruce de variables interés en la actividad turística $x$ dedicación

\begin{tabular}{lccc} 
& Valor & gl & \multicolumn{2}{c}{ Significación asintótica (bilateral) } \\
\hline Chi-cuadrado de Pearson & $9,127^{\text {a }}$ & 4 &, 068 \\
Razón de verosimilitud & 8,412 & 4 & \\
N de casos válidos & 386 & &
\end{tabular}

Nota: a. 3 casillas $(33,3 \%)$ han esperado un recuento menor que 5. El recuento mínimo esperado es 1,00.

Fuente: Elaboración propia, 2020.

Tal como se aprecia en la Tabla 8 , el $74 \%$ de la población indistintamente del nivel de estudio, dedicaría tiempo parcial $(59,8 \%)$ o completo $(14,2 \%)$ durante la semana a actividades relacionadas con el turismo rural en el municipio. Con dedicación parcial, profesionales $(58,3 \%)$, técnicos $(61,1 \%)$ y para quienes cuenta con formación básica o secundaría $(55,4 \%)$. Con relación a dedicación completa, se tendría disposición en profesionales $(14,2 \%)$, técnicos $(11,1 \%)$ y de educación primaria o bachilleres $(15,4 \%)$. 
Tabla 8

Tabla cruzada interés en la actividad turística $\mathbf{x}$ nivel de estudio

\begin{tabular}{|c|c|c|c|c|c|c|}
\hline & & & Nivel_Estud & & & \\
\hline & & & $\begin{array}{l}\text { Básica- } \\
\text { Secundaria }\end{array}$ & Técnico & Profesional & Total \\
\hline \multirow{6}{*}{$\begin{array}{l}\text { Si es de su interés } \\
\text { desarrollar } \\
\text { actividades } \\
\text { relacionadas con el } \\
\text { turismo rural, ¿qué } \\
\text { tiempo dedicaría? }\end{array}$} & \multirow{2}{*}{$\begin{array}{l}\text { No estoy } \\
\text { interesado }\end{array}$} & Recuento & 19 & 70 & 11 & 100 \\
\hline & & $\begin{array}{l}\% \text { dentro de Nivel_ } \\
\text { Estudio }\end{array}$ & $29,2 \%$ & $24,6 \%$ & $30,6 \%$ & $25,9 \%$ \\
\hline & \multirow{2}{*}{$\begin{array}{l}\text { Fines de } \\
\text { semana y } \\
\text { festivos }\end{array}$} & Recuento & 36 & 174 & 21 & 231 \\
\hline & & $\begin{array}{l}\text { \% dentro de Nivel_ } \\
\text { Estudio }\end{array}$ & $55,4 \%$ & $61,1 \%$ & $58,3 \%$ & $59,8 \%$ \\
\hline & \multirow{2}{*}{$\begin{array}{l}\text { De lunes a } \\
\text { domingo }\end{array}$} & Recuento & 10 & 41 & 4 & 55 \\
\hline & & $\begin{array}{l}\% \text { dentro de Nivel_ } \\
\text { Estudio }\end{array}$ & $15,4 \%$ & $14,4 \%$ & $11,1 \%$ & $14,2 \%$ \\
\hline \multirow[b]{2}{*}{ Total } & & Recuento & 65 & 285 & 36 & 386 \\
\hline & & $\begin{array}{l}\% \text { dentro de Nivel_ } \\
\text { Estudio }\end{array}$ & $100,0 \%$ & $100,0 \%$ & $100,0 \%$ & $100,0 \%$ \\
\hline
\end{tabular}

Fuente: Elaboración propia, 2020.

Aproximadamente, un cuarto de la población (ver Tabla 8) se muestra no interesada en participar del desarrollo de las actividades turísticas, indistintamente del nivel de formación, de esta manera se evidencia que no existe correspondencia entre el interés en las actividades turísticas y su nivel de formación; reflejado de igual forma en la Tabla 9, con los valores de Chi-cuadrado de 1,387 y razón de verosimilitud de igual valor, y finalmente, el nivel de significancia de 0,846 .

Tabla 9

Pruebas Chi-cuadrado cruce de variables interés en la actividad turística $\mathbf{x}$ nivel de estudio

\begin{tabular}{lccc}
\hline & Valor & gl & Significación asintótica (bilateral) \\
\hline Chi-cuadrado de Pearson & $1,387^{\mathrm{a}}$ & 4 &, 846 \\
Razón de verosimilitud & 1,387 & 4 &, 846 \\
N de casos válidos & 386 & & \\
\hline
\end{tabular}

Nota: a. 0 casillas $(0,0 \%)$ han esperado un recuento menor que 5 . El recuento mínimo esperado es 5,13.

Fuente: Elaboración propia (2020). 
En relación con el interés de la población en participar del ejercicio de la actividad turística en el municipio, de acuerdo con el lugar de asentamiento, se destaca en la Tabla 10, la intención de los habitantes de la zona de dedicar tiempo a realizar actividades ligadas a la promoción de turismo en tiempo parcial, fines de semana y festivos $(91,7 \%)$, razonable al considera que durante la semana ocuparían su tiempo a las actividades propias del campo.

Tabla 10

Tabla cruzada en la actividad turística $x$ zona donde habita

\begin{tabular}{|c|c|c|c|c|c|}
\hline & & & \multicolumn{2}{|c|}{ Zona donde habita } & \multirow[b]{2}{*}{ Total } \\
\hline & & & $\begin{array}{l}\text { Población } \\
\text { casco } \\
\text { urbano del } \\
\text { municipio }\end{array}$ & $\begin{array}{l}\text { Población } \\
\text { área rural del } \\
\text { municipio }\end{array}$ & \\
\hline \multirow{6}{*}{$\begin{array}{l}\text { Si es de su interés } \\
\text { desarrollar } \\
\text { actividades } \\
\text { relacionadas con el } \\
\text { turismo rural, ¿qué } \\
\text { tiempo dedicaría? }\end{array}$} & \multirow{2}{*}{ No estoy interesado } & Recuento & 99 & 1 & 100 \\
\hline & & $\begin{array}{l}\% \text { dentro de Zona } \\
\text { donde habita }\end{array}$ & $26,5 \%$ & $8,3 \%$ & $25,9 \%$ \\
\hline & \multirow{2}{*}{$\begin{array}{l}\text { Fines de semana y } \\
\text { festivos }\end{array}$} & Recuento & 220 & 11 & 231 \\
\hline & & $\begin{array}{l}\% \text { dentro de Zona } \\
\text { donde habita }\end{array}$ & $58,8 \%$ & $91,7 \%$ & $59,8 \%$ \\
\hline & \multirow[b]{2}{*}{ De lunes a domingo } & Recuento & 55 & 0 & 55 \\
\hline & & $\begin{array}{l}\% \text { dentro de Zona } \\
\text { donde habita }\end{array}$ & $14,7 \%$ & $0,0 \%$ & $14,2 \%$ \\
\hline \multirow{2}{*}{\multicolumn{2}{|c|}{ Total }} & Recuento & 374 & 12 & 386 \\
\hline & & $\begin{array}{c}\% \text { dentro de Zona } \\
\text { donde habita }\end{array}$ & $100,0 \%$ & $100,0 \%$ & $100,0 \%$ \\
\hline
\end{tabular}

Fuente: Elaboración propia, 2020.

En contraste la población que habita en el casco urbano del municipio, demuestra principalmente su intención en dedicar tiempo durante la semana $(73,5 \%)$ a las actividades turísticas; y de estas en mayor proporción fines de semana $(58,8 \%)$. Existe, sin embargo, desinterés por una parte de la población, llamando la atención que principalmente se centra en la que habita en el casco urbano, dejando en evidencia que para la población del área rural, tendría mayores expectativas frente a los beneficios esperados como resultados del ejercicio del turismo rural en el municipio.
En cuanto al potencial del turismo rural como alternativa de desarrollo local del municipio, se reconfirma, en la Tabla 11, el interés y percepción de la población rural frente a las oportunidades del ejercicio del turismo rural como alternativa de desarrollo del municipio (100\%); asimismo, el casco urbano tiene una gran aceptación frente a la apuesta de este tipo de actividades turísticas y su contribución a la mejora de vida de sus habitantes $(94,1 \%)$. 
Tabla 11

Tabla cruzada potencial actividad turística $x$ zona donde habita

\begin{tabular}{|c|c|c|c|c|c|}
\hline & & & \multicolumn{3}{|c|}{ Zona donde habita } \\
\hline & & & $\begin{array}{l}\text { Población } \\
\text { casco } \\
\text { urbano del } \\
\text { municipio }\end{array}$ & $\begin{array}{l}\text { Población } \\
\text { área rural del } \\
\text { municipio }\end{array}$ & Total \\
\hline \multirow{6}{*}{$\begin{array}{l}\text { Cree usted que si se } \\
\text { potenciara el turismo } \\
\text { rural en el municipio, esto } \\
\text { contribuiría al desarrollo de } \\
\text { la comunidad, por ejemplo } \\
\text { generando; mayor cantidad } \\
\text { de empleos, cuidado del } \\
\text { ambiente, mejoramiento } \\
\text { en la infraestructura (vías, } \\
\text { servicios e instalación) }\end{array}$} & \multirow[b]{2}{*}{ No } & Recuento & 4 & 0 & 4 \\
\hline & & $\begin{array}{l}\% \text { dentro de Zona } \\
\text { donde habita }\end{array}$ & $1,1 \%$ & $0,0 \%$ & $1,0 \%$ \\
\hline & \multirow[b]{2}{*}{ Medianamente } & Recuento & 18 & 0 & 18 \\
\hline & & $\begin{array}{l}\% \text { dentro de Zona } \\
\text { donde habita }\end{array}$ & $4,8 \%$ & $0,0 \%$ & $4,7 \%$ \\
\hline & \multirow[b]{2}{*}{$\mathbf{S i}$} & Recuento & 352 & 12 & 364 \\
\hline & & $\begin{array}{l}\% \text { dentro de Zona } \\
\text { donde habita }\end{array}$ & $94,1 \%$ & $100,0 \%$ & $94,3 \%$ \\
\hline \multirow[b]{2}{*}{ Total } & & Recuento & 374 & 12 & 386 \\
\hline & & $\begin{array}{l}\% \text { dentro de Zona } \\
\text { donde habita }\end{array}$ & $100,0 \%$ & $100,0 \%$ & $100,0 \%$ \\
\hline
\end{tabular}

Fuente: Elaboración propia, 2020.

Los resultados expuestos reflejan la situación actual del municipio de Santo Tomás frente al ejercicio de actividades de turismo rural, desde la perspectiva de los actores estratégicos, los más involucrados en el tema y de la población local en general. El escenario actual, involucra varios aspectos, como socioeconómicos, institucionales y ambientales, esto acorde a lo que plantean Morales, et al. (2015); y, Loor, et al. (2021), quienes señalan que el turismo rural es quizá una de las prácticas humanas que abarca el mayor número de interrelaciones, puesto que tiene un papel en la dimensión económica, al generar empleo, ingresos y desarrollar mercado de servicios conexos; en la dimensión social, en lo que tiene que ver con el desarrollo artístico, la recreación, valoración y preservación de las herencias culturales.

En la dimensión ambiental, algunas actividades turísticas se fundamentan en la administración y gestión de recursos naturales, en el manejo equilibrado de ecosistemas y preservación de la diversidad biológica; $\mathrm{y}$ en la dimensión institucional, desde el fortalecimiento de la capacidad de realizar autoanálisis para la toma de decisiones que impacten la comunidad. Por otra parte, se encuentra la dimensión física, con base en lo señalado por Dirven, et al. (2011), quienes plantean la importancia de este aspecto toda vez que, al mismo tiempo, e igualdad de atractivos turísticos, las inversiones tienden a concentrarse en las regiones mejor dotadas de infraestructura (en cantidad y calidad).

\subsection{Parte: Propuesta del nuevo modelo logístico para el aprovechamiento del potencial turístico del municipio Santo Tomás- Atlántico en Colombia.}

Partiendo de la revisión de los diversos modelos estudiados y del trabajo 
de campo realizado, se presenta la siguiente propuesta del nuevo modelo logístico para el aprovechamiento del potencial turístico del municipio Santo Tomás-Atlántico basándose en los planteamientos de Correa, et al. (2017), tal como se muestra en la Figura I.

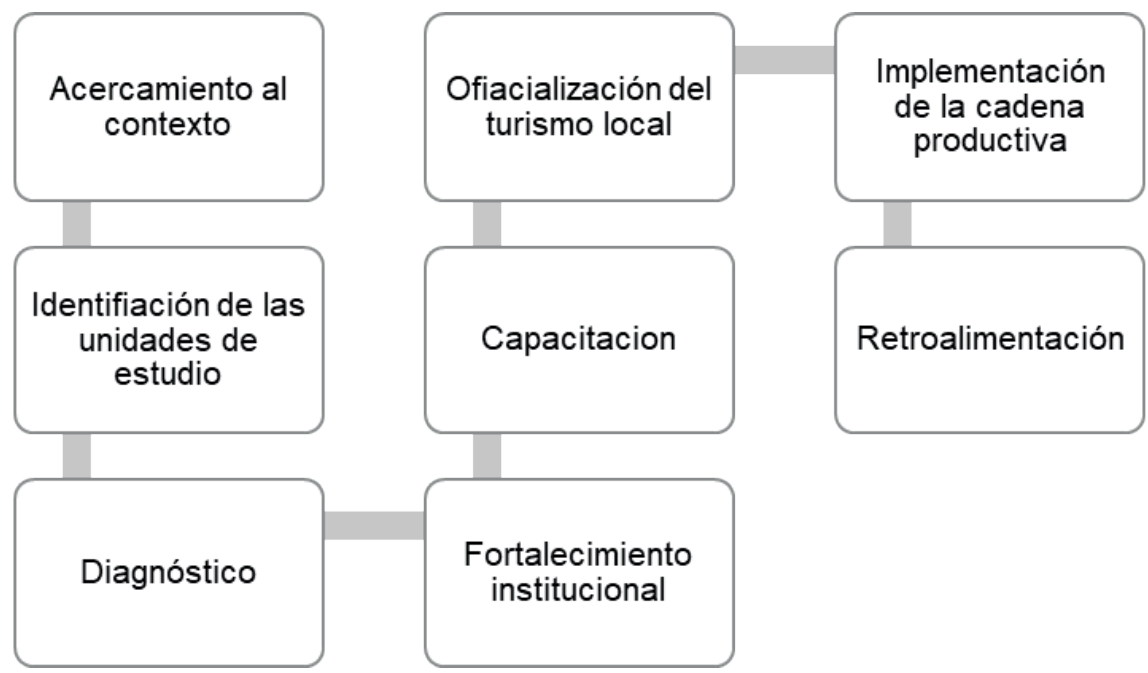

Fuente: Elaboración propia, 2020

Figura I: Pasos para la puesta en marcha del modelo logístico

Con base en la Figura anterior, se destaca que la propuesta logística para el aprovechamiento del potencial turístico del municipio Santo Tomás-Atlántico, se desarrolla con un primer paso que se direcciona hacia el acercamiento, el cual implica los primeros reconocimientos del entorno, con el fin de comenzar a definir las áreas de trabajo. Seguidamente, se definen las unidades de estudio que serán medidas en el diagnóstico, proceso que implica los siguientes elementos: a) Dimensión económica, b) desde la dimensión institucional, c) con respecto a la dimensión social, y d) en cuanto a la dimensión física. Por medio del proceso diagnóstico, se pasan a las siguientes fases que se vinculan hacia:

1. Fortalecimiento institucional: Para lograr que los entes públicos brinden un mayor acompañamiento y vigilancia de los procesos turísticos de este municipio.

2. Capacitación: Para formar a los ciudadanos en las labores que les permitan explotar el potencial turístico del municipio y hacer de ello un negocio sostenible.

3. Oficialización del turismo local: La creación de toda la documentación reglamentaria y la definición de los diversos cargos, brinda una importante ventaja competitiva.

Se debe destacar que para la generación de un organigrama de este tipo de organizaciones de economía solidaria, se requiere de la participación democrática de sus miembros, por lo que basándose en ello, la estructura adecuada seria la que se aprecia en la Figura II. 


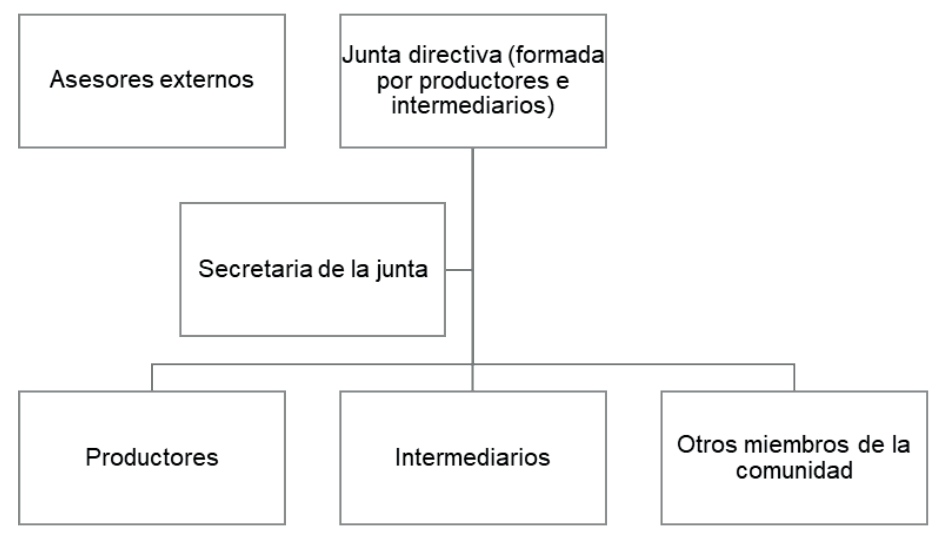

Fuente: Elaboración propia, 2020.

\section{Figura II: Estructura del modelo propuesto}

Con base a este nuevo organigrama, se presenta una clara cohesión entre los tres actores principales; los productores primarios, los intermediarios y los miembros de la comunidad. Dicho esto, la fase de implantación del modelo logístico se basa en los siguientes seis ejes que se desarrollan de manera cíclica, como se observan en la Figura III.

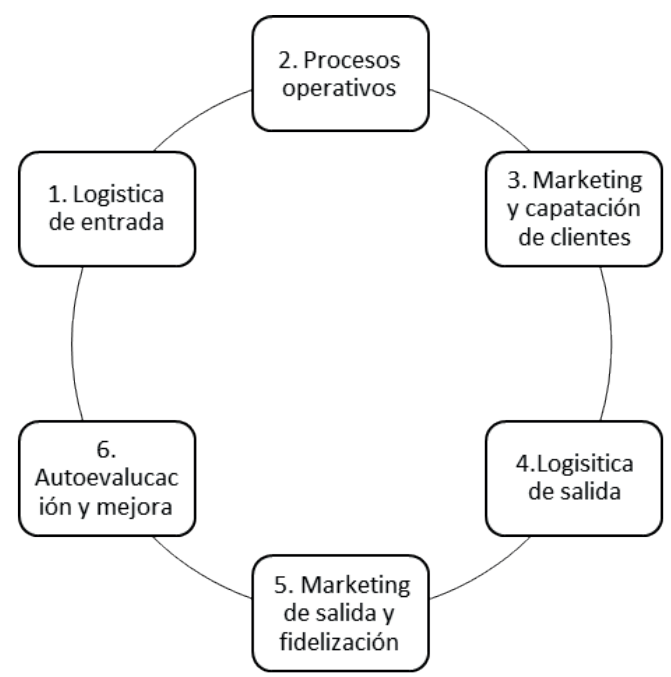

Fuente: Elaboración propia, 2020.

Figura III: Ciclo del modelo logístico 
Al observar la Figura III, se aprecia como los seis ejes del modelo logístico se integran entre sí, de manera que el primer paso de logística, de entrada permita sedimentar las bases para las siguientes fases; cerrando con la logística de salida y el marketing de salida, donde se busca una fidelización del consumidor. El último paso del modelo planteado, se direcciona con la autoevaluación, de modo que el mismo sistema constantemente pueda ser mejorado, con la finalidad de responder a las necesidades y exigencias del mercado y los consumidores, para así posicionar el sector turístico del municipio.

\section{Conclusiones}

En respuesta a la pregunta planteada en esta investigación, se logró caracterizar el municipio de Santo Tomás-Atlántico (Colombia) desde su potencial en turismo rural, identificando las condiciones actuales del territorio y las condiciones para que esta actividad turística pueda ejercerse acorde a su realidad social, cultural y de infraestructura física y natural.

En el municipio de Santo Tomás, la dimensión económica desarrollada desde el turismo rural es muy escasa, debido a que esta actividad, pese a tener un número importante de elementos positivos, como lo es la belleza escénica y recursos naturales, no ha sido mayormente explotada, por lo que la generación de ingresos proveniente de ella es precaria. No obstante, el mayor incentivo de las personas dispuestas a desarrollar turismo rural, se relaciona con la alternativa de ser una fuente de generación de beneficios económicos, representada en una actividad complementaria.

Desde la dimensión institucional, existen falencias al no encontrarse incluido por lo menos en el ámbito local dentro del Plan de Desarrollo del Municipio 2016-2019 y en los planes de Gobierno proyectados a partir de 2020, la promoción o fomento de la actividad turística del municipio, inclusive como fuente de generación de ingresos, lo cual dificulta aún más su impulso y desarrollo. El Gobierno local, es un agente clave para el desarrollo del turismo rural, y si bien la existencia de una Política Nacional de Turismo, que puede generar expectativas para pensar en el crecimiento de partidas desde el orden nacional, debe mantener un rol protagónico en el desarrollo del turismo. Santo Tomás no ha asumido por su parte, el rol fundamental que le compete en el desarrollo de la actividad turística de manera integral, hasta el momento estas actividades son vistas solo de forma estacional a través de las festividades y tradiciones del municipio.

Con respecto a la dimensión social, la población si bien manifiesta en mayor parte interés por desarrollar la actividad turística, en la realidad esta no se lleva a cabo o no tienen claridad sobre la misma. Se evidenció en una porción importante de la población, incluso sus gobernantes, desconocimiento sobre el tema, e incluso sobre el significado de "turismo rural" y los términos asociados a esta actividad. Lo anterior es importante pues el estudio reciente de Lo, Chin y Law (2019), muestra que el respaldo de la comunidad rural es, por ejemplo, un moderador entre la calidad de la estadía y la competitividad de los servicios de turismo rural; con lo cual, en caso de desarrollarse este tipo de turismo en el municipio de Santo Tomás, el respaldo de la comunidad puede generar ventajas competitivas.

En cuanto a la dimensión física, la infraestructura turística puede considerarse aceptable dentro del casco urbano. Sin embargo, es notorio que es insuficiente en la zona rural (campo, fincas y humedales), quedando desprovistas de estos servicios, inclusive servicios básicos como energía y acceso a agua, esto dificultaría el desarrollo de la actividad turística por parte de la población rural.

Una de las mayores limitaciones, es la falta de programas y planes de capacitación, además la ausencia de organizaciones ligadas al turismo rural, que podrían como mecanismo de cooperación entre el sector público y privado, aunar esfuerzos voluntariamente en búsqueda del ejercicio de la actividad 
turística en el municipio. El nuevo modelo logístico, integra una serie de pasos para su aplicación, acompañado de seis ejes de actividad, que fundamentándose en el trabajo colaborativo entre todas las partes del proceso y la comunidad, busca generar un importante cambio dentro del desarrollo del potencial turístico del municipio de Santo Tomas.

Finalmente, es posible concluir entonces que el municipio de Santo Tomás (Atlántico) cuenta con vocación turística para desarrollar la actividad en el territorio, sustentada en paisajes con belleza escénica y alternativas de diversas actividades en ellas, además de las ya desarrolladas en el territorio, y que depende del esfuerzo mancomunado de sus habitantes así como entes gubernamentales.

\section{Referencias bibliográficas}

Ascón, J., y Argibay, A. (2020). Herramienta para el desarrollo de metahabilidades directivas en jefes de Recepción de hotel. SUMMA. Revista Disciplinaria en Ciencias Económicas y Sociales, 2(2), 61-82.

Constabel, S., Oyarzun, E., y Szmulewicz, P. (2008). Agroturismo en Chile: Caracterización y perspectivas. Ediciones de la Universidad Austral de Chile y Fundación para la Innovación Agraria.

Correa, M., Hernández, J. C., y Loredo, N. (2017). La cadena productiva del ecoturismo como contribución al desarrollo local. Retos de la Dirección, 11(1), 147-171.

Departamento Administrativo Nacional de Estadística-DANE (2016). Colombia, estimaciones de la migración. 19852005 y proyecciones 2005-2020. Nacionales y departamentales. https://www.dane.gov.co/index.php/ estadisticas-por-tema/demografia-ypoblacion/movilidad-y-migracion
Diaz-Christiansen, S., Pérez-Gálvez, J. C., y Ortega, M. (2017). Análisis de las motivaciones hacia el ecoturismo en Ecuador. Un estudio de caso en la Isla Santay. Revista Turydes: Turismo y Desarrollo, (22), 1-18. https://www. eumed.net/rev/turydes/22/isla-santay. html

Dirven, M., Echeverri, R., Sabalain, C., Rodríguez, A., Candia, D., Peña, C., y Faiguenbaum, S. (2011). Hacia una nueva definición de "rural" con fines estadísticos en América Latina. Colecciones de la CEPAL. Naciones Unidas.

Faiguenbaum, S. (2011). Definiciones oficiales de "rural" y/o "urbano" en el mundo. En M. Dirven, R. Echeverri, C. Sabalain, A, Rodríguez, D. Candia, C. Peña y S. Faiguenbaum, Hacia una nueva definición de "rural" con fines estadísticos en América Latina (pp. 67-90). Colecciones de la CEPAL. Naciones Unidas.

Gadotti, S. J., y Stock, L. T. (2009). Logística integrada de eventos: El caso del Festival de Danza de Joinville (SC) - Brasil. Estudios y Perspectivas en Turismo, 18(3), 302-317.

Gallardo, G. (2009). Levantamiento del inventario de atractivos turísticos del Departamento del Atlántico. Editorial de la Universidad Autónoma del Caribe.

Gobernación del Atlántico Colombia (2016). Plan de Desarrollo 2016 - 2019. Atlántico Líder. https:// www.atlantico.gov.co/images/ stories/plan desarrollo/plan de desarrollo 2016 2016 definitivo.pdf

Lo, M-C., Chin, C-H., y Law, F-Y. (2019). Tourists' perspectives on hard and soft services toward rural tourism destination competitiveness: Community support as a moderator. Tourism and Hospitality Research, 
Pizarro, Ana Barrera; Alonso Ballestas y Marlene Ramírez, Javier

Modelo logístico para el aprovechamiento del potencial turístico del municipio Santo TomásAtlántico en Colombia

19(2), 139-157. $\quad$ https://doi. org/10.1177/1467358417715677

Loor, L., Plaza, N., y Medina, Z. (2021). Turismo comunitario en Ecuador: Apuntes en tiempos de pandemia. Revista de Ciencias Sociales (Ve), XXVII(1), 265-277.

Martín, J. M., Salinas, J. A., Rodríguez, J. A., y Jiménez, J. D. D. (2017). Assessment of the tourism's potential as a sustainable development instrument in terms of annual stability: Application to Spanish rural destinations in process of consolidation. Sustainability (Switzerland), 9(10). https://doi. org/10.3390/su9101692

Martínez, J., y Padilla, L. (2020). Innovación organizacional y competitividad empresarial: Centros estéticos de turismo de salud en Cali-Colombia. Revista de Ciencias Sociales (Ve), XXVI(2), 120-132. https://doi. org/10.31876/rcs.v26i2.32428

Ministerio de Comercio, Industria y Turismo MinCIT (2012a). Diagnóstico Turismo de Naturaleza en Colombia. MinCIT. T\&L - AVIAEXPORT https://www. colombiaproductiva.com/CMSPages/ GetFile.aspx?guid $=56 \mathrm{e} 56497-881 \mathrm{~d}-$ 41f6-b23c-4df2d0685f6b

Ministerio de Comercio, Industria y Turismo - MinCIT (2012b). Política de turismo de naturaleza. Desarrollo turístico en Colombia. MinCIT. http://www.bogotaturismo.gov. co/sites/intranet.bogotaturismo. gov.co/files/POLITICA\%20 DE $\% 20$ TURISMO\%20DE\%20 NATURALEZA.pdf
Morales, L. A., Cabral, A., Aguilar, A., Velazco, L., y Holguin, O. (2015). Agroturismo y competitividad, como oferta diferenciadora: el caso de la ruta agrícola de San Quintín, Baja California. Revista Mexicana de Agronegocios, 37, 185-196. https:// doi.org/10.22004/ag.econ.226159

Niebles, L. D., Santamaria, A. E., y Niebles, W. A. (2018). Las habilidades gerenciales $\mathrm{y}$ el liderazgo transformacional como escenario de fortalecimiento organizacional en las Mipymes turísticas de Barranquilla - Colombia. En J. A. Navarro (Ed.), Herramientas gerenciales para la sostenibilidad de las organizaciones de la Costa Caribe Colombiana (pp. 1-29). Corporación Universitaria Latinoamericana.

Nugra, M. A., Illescas, W. H., Cuadros, P. A., y Valdivia, R. A. (2021). Turismo minero en Yanacocha: Una alternativa de desarrollo para la región de Cajamarca-Perú. Revista de Ciencias Sociales (Ve), XXVII(1), 278-289.

Organización Mundial del Turismo - OMT (17 de enero de 2017). El turismo internacional mantiene un crecimiento sostenido pese a las dificultades. UNWTO. https://www.unwto.org/es/ archive/press-release/2017-01-17/elturismo-internacional-mantiene-uncrecimiento-sostenido-pese-las-dificul

Spiegel, M. R., y Stephen, L. J. (2005). Estadística. McGraw-Hill.

Zúñiga-Collazos, A. (2015). Análisis de la investigación y desarrollo turístico en Colombia. Revista Espacios, 36(18), 9. 